\title{
Diagnostic performance of conventional endoscopy in the identification of submucosal invasion by early gastric cancer: the "non-extension sign" as a simple diagnostic marker
}

\author{
Takashi Nagahama ${ }^{1} \cdot K^{K e n s h i ~} \mathrm{Yao}^{2} \cdot$ Kentaro Imamura $^{3}$ - Toshiki Kojima ${ }^{2} \cdot$ \\ Kensei Ohtsu ${ }^{1} \cdot$ Kenta Chuman $^{3} \cdot$ Hiroshi Tanabe ${ }^{3} \cdot$ Rino Yamaoka $^{1}$. \\ Akinori Iwashita ${ }^{3}$
}

Received: 1 December 2015/Accepted: 23 April 2016/Published online: 10 May 2016

(C) The International Gastric Cancer Association and The Japanese Gastric Cancer Association 2016

\begin{abstract}
Background and aim The ability to differentiate between mucosal (M) or microinvasive submucosal (SM1: depth of less than $500 \mu \mathrm{m}$ ) and invasive submucosal (SM2: depth of $500 \mu \mathrm{m}$ or more) cancer is paramount when choosing the method of treatment for early gastric cancer (EGC). The "non-extension sign" relates to a localized increase in thickness and rigidity due to massive submucosal invasion by a cancer. The present study sought to assess the ability of conventional endoscopy (CE) to correctly identify SM2 cancer using only the non-extension sign.

Methods This is a retrospective study based on a prospectively collected database. EGCs had been diagnosed according to invasion depth as M-SM1 or SM2. In terms of the endoscopic diagnostic criterion, lesions positive for the non-extension sign were classified as SM2 cancers, while those negative for the non-extension sign were classified as M-SM1 cancers. Histopathological findings were used as the gold standard.

Results We examined a total of 863 lesions from 704 patients, comprising 104 true-positive, 733 true-negative, 9 false-positive, and 17 false-negative lesions. This yielded a sensitivity of $92.0 \%$ (95\% confidence interval (CI), $87.0-97.0 \%)$, a specificity of $97.7 \% \quad(95 \% \quad \mathrm{CI}$,
\end{abstract}

Takashi Nagahama

nagahamagogo@gmail.com

1 Department of Gastroenterology, Fukuoka University Chikushi Hospital, 1-1-1 Zokumyoin, Chikushino City, Fukuoka 818-8502, Japan

2 Department of Endoscopy, Fukuoka University Chikushi Hospital, Fukuoka, Japan

3 Department of Pathology, Fukuoka University Chikushi Hospital, Fukuoka, Japan
96.7-98.8\%), a positive predictive value of $85.9 \%$ (95\% CI, 79.7-92.1\%), a negative predictive value of $98.8 \%$ (95\% CI, 98.0-99.6\%), and a diagnostic accuracy of $96.9 \%$ (95\% CI, 95.8-98.1\%).

Conclusion The non-extension sign may be useful for accurately determining the suitability of minimally invasive endoscopic treatment. Nevertheless, considering the limitations of retrospective analysis, a further prospective study is warranted to confirm the diagnostic reliability of the non-extension sign.

Keywords Submucosal invasion - Early gastric cancer . Endoscopic submucosal dissection - Conventional endoscopy $\cdot$ Non-extension sign

\section{Introduction}

The advent of endoscopic submucosal dissection (ESD) has enabled en bloc resection of large early gastric cancer (EGC), and has expanded the indications for endoscopic therapy $[1,2]$.

The risk of lymph node metastasis is reported to be extremely low for the following types of EGC: differentiated intramucosal (M) cancers of any size with no ulceration; differentiated superficial submucosal (SM1, depth of less than $500 \mu \mathrm{m}$ ) cancers which have a diameter $\leq 30 \mathrm{~mm}$; and undifferentiated $\mathrm{M}$ cancers with no ulceration and a diameter $\leq 20 \mathrm{~mm}$. Endoscopic treatment can therefore be curative in these three groups [1-3]. Accordingly, when determining the treatment plan for patients with gastric cancer, it is very important to be able to differentiate between M-SM1 and deeper submucosal (SM2, depth of $500 \mu \mathrm{m}$ or more) cancers when attempting to correctly distinguish between patients who require open surgical 
resection and those for whom less invasive ESD can be curative.

However, few studies have examined the diagnostic performance of $\mathrm{CE}$, the most widely used modality, when determining the depth of EGC invasion. In previous studies, determination of the depth of invasion has been based on the experience of the endoscopist, using a number of different indices of depth of invasion as derived from statistical analyses of the morphological characteristics of SM2 cancers [4-7]. Since the morphology of EGCs is readily altered by the degree by which the gastric wall is extended by insufflated air, this diagnostic marker lacks both objectivity and reproducibility. However, previous reports have not recorded the conditions under which this diagnostic marker was observed.

Yao et al. reported that when they outlined the histologically confirmed SM2-invasive area of an EGC on the macroscopic image of the resected specimen, and then retrospectively identified the corresponding SM2 invasive area on endoscopic images, a specific "nonextension sign" became clearly visible [8]. This nonextension sign is caused by the invasion of cancer cell nests and/or a desmoplastic reaction as far as the SM2 level, thereby causing localized thickening and rigidity. When the gastric wall is strongly extended by endoscopic insufflation of a large volume of air, the SM2invasive area can be seen as a trapezoid elevation (Fig. 1).

The present study sought to determine the diagnostic ability of CE to correctly identify SM2 cancer using the non-extension sign as the only diagnostic criterion.

\section{Materials}

\section{Sampling of study subjects}

Sampling from a pathology database

(1) A list was created of all gastric cancers endoscopically or surgically resected at Fukuoka University Chikushi Hospital from January 2006 to December 2012 from a pathological diagnosis database

(2) Next, cases of advanced cancer (including advanced gastric cancer simulating early cancer, namely type 0 advanced cancer) were excluded from this list and only lesions histopathologically diagnosed as EGC were sampled

(3) Lesions where a determination of invasion depth was histopathologically difficult were further excluded

\section{Sampling from an endoscopy database}

(1) All histopathologically sampled EGC cases that had not been subjected to preoperative examination at our hospital were excluded, with reference to an endoscopy database

(2) In addition, cases described as undiagnosable due to poor observation conditions on the basis of records from the endoscopy database, such as infeasibly strong extension of the gastric wall due to a gag reflex or an inability to make observations due to bleeding from the lesion, were excluded

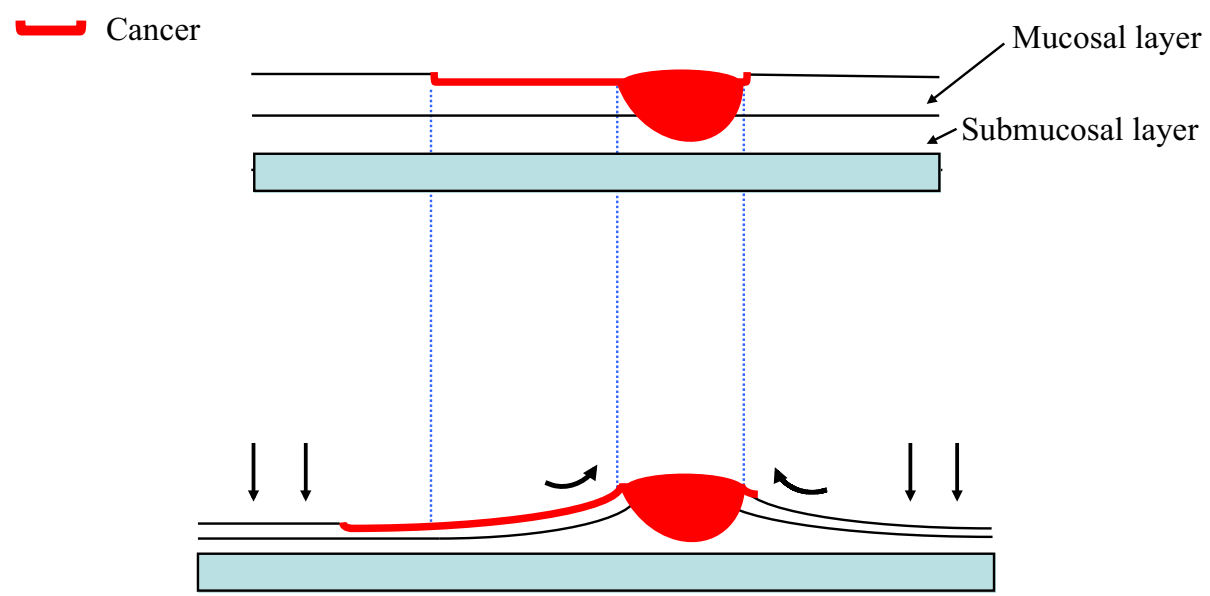

Fig. 1 Morphological changes in areas of intramucosal cancer and SM-invasive areas with extension of the gastric wall. With endoscopic insufflation of a large volume of air, the noncancerous mucosa and areas of intramucosal cancer become flattened and extended. In contrast, the SM2-invasive area can be seen as a trapezoid elevation with elevation of the surrounding mucosa due to localized thickening and hardening associated with submucosal invasion by cancer cells 


\section{Methods}

In this study, cases of EGC that were prospectively diagnosed by endoscopy were accumulated and retrospectively analyzed for invasion depth diagnostic accuracy.

\section{Endoscopic procedure for determining the submucosal invasion of early gastric cancers}

\section{Premedication and sedation}

All patients underwent optimum preparation, drinking a mixture of mucolytic and defoaming agents in water $30 \mathrm{~min}$ before the procedure. The formula was $20,000 \mathrm{U}$ pronase (Kaken Pharmaceutical, Tokyo, Japan), $1 \mathrm{~g}$ sodium bicarbonate, and $10 \mathrm{~mL}$ dimethylpolysiloxane (20 mg/mL; Horii Pharmaceutical, Osaka, Japan) in $100 \mathrm{~mL}$ water. One milliliter of scopolamine butylbromide (20 mg/mL; Boehringer Ingelheim, Tokyo, Japan) or $1 \mathrm{mg}$ of glucagon (Eisai, Tokyo, Japan) was routinely administered as an antiperistaltic agent. Most patients underwent sedation, with 3-10 $\mathrm{mg}$ of diazepam $(5 \mathrm{mg} / \mathrm{mL}$; Takeda Pharmaceutical, Tokyo, Japan) injected into the patient intravenously.

\section{Endoscopes}

All procedures were performed using an Evis Lucera Spectrum system (Olympus, Tokyo, Japan), with a highresolution endoscope (GF-Q240, GIF-Q240Z, GIF-H260 or GIF-H260Z; Olympus).

\section{Diagnostic criterion for SM2: definition of the "non- extension sign"}

We used only the non-extension sign under the unified viewing method as a marker for the endoscopic diagnosis of invasion defined as SM2 or more. This non-extension sign can only be seen when the entire stomach wall is strongly distended through insufflation of a large volume of air.

When the stomach wall is strongly distended through insufflation of a large volume of air, M-SM1 cancers and the noncancerous mucosa surrounding an SM2-invasive cancer will be extended laterally and become flattened. In contrast, two morphological characteristics become clearly visible with SM2 cancers: (1) the SM2-invasive area is not extended and forms a trapezoid elevation (Figs. 2b, 3c); and (2) mucosal folds converge and become elevated at the SM2-invasive area (Fig. 3b, c) [8]. Lesions were assessed as positive for the non-extension sign when both or either of these findings were identified. If neither of these findings was present, the lesion was assessed as negative for the non-extension sign.

Even for M-SM1 cancers, fold convergence is seen in lesions associated with an ulcer scar, presenting an appearance similar to the non-extension sign (Fig. 4a). In ulcer scars, because the level of inflammatory fibrosis in the submucosa gradually becomes coarser upon moving from the center of the scar to its periphery, localized thickenings do not form within the submucosal layer. Accordingly, no localized trapezoid elevation is formed, and the converging mucosal folds follow straight paths as they converge at the center of the ulcer scar, with no elevation (Fig. 4b, c) [8]. Morphologies of this type were assessed as negative for the non-extension sign.

\section{Examination methods}

Using conventional white-light imaging endoscopy and chromoendoscopy (staining with $0.1 \%$ indigo carmine), we determined the lateral margins of invasion by EGCs, and then determined the depth of invasion.

First, we slowly insufflated air to strongly extend the gastric wall (Figs. 2a, b, 3a, b, 4a, b). Strong extension of the gastric wall is defined as extension that meets one or both of the following criteria: (1) full extension of the folds on the greater curvature of the gastric corpus; (2) extension until blood vessels surrounding the EGC can be clearly delineated (Figs. 2a, b, 3a, b). All endoscopists determined the presence or absence of the non-extension sign based on these criteria for strong extension of the gastric wall.

Next, we observed the lesion from an oblique or tangential direction and determined whether the non-extension sign was present. Lesions positive for the non-extension sign were diagnosed as SM2 (Figs. 2b, 3c), while lesions negative for the non-extension sign were diagnosed as M-SM1 (Fig. 4c).

Each endoscopic examination was performed by a single independent endoscopist, with the diagnosis recorded in a database immediately following each procedure. For the purposes of data collection, all endoscopists recorded the presence or absence of the non-extension sign and the determined invasion depth in the database.

When diagnosis was impossible because observations could not be made due to bleeding from the lesion or because it was not possible to strongly extend the gastric wall due to a gag reflex or other impediment, the endoscopist recorded these findings in the endoscopy database. The 16 endoscopists who participated in this study were all accredited by the Japan Gastroenterological Endoscopy Society to perform endoscopic examinations. 
Fig. 2 a 0-IIa + IIc type SM2 cancer. Endoscopic findings with insufflation of a moderate volume of air into the stomach. b Classical non-extension sign. With strong extension of the gastric wall through endoscopic insufflation of a large volume of air, there is full extension of the folds in the greater curvature of the gastric corpus, and the blood vessels surrounding the EGC can be clearly delineated. The SM2-invasive area is not extended and forms a trapezoid elevation; mucosal folds

converge and become elevated at the SM2-invasive area (yellow arrows). c Histological findings. Invasion of the submucosa by a large number of cancer cells is evident. SM2 depth $2000 \mu \mathrm{m}$
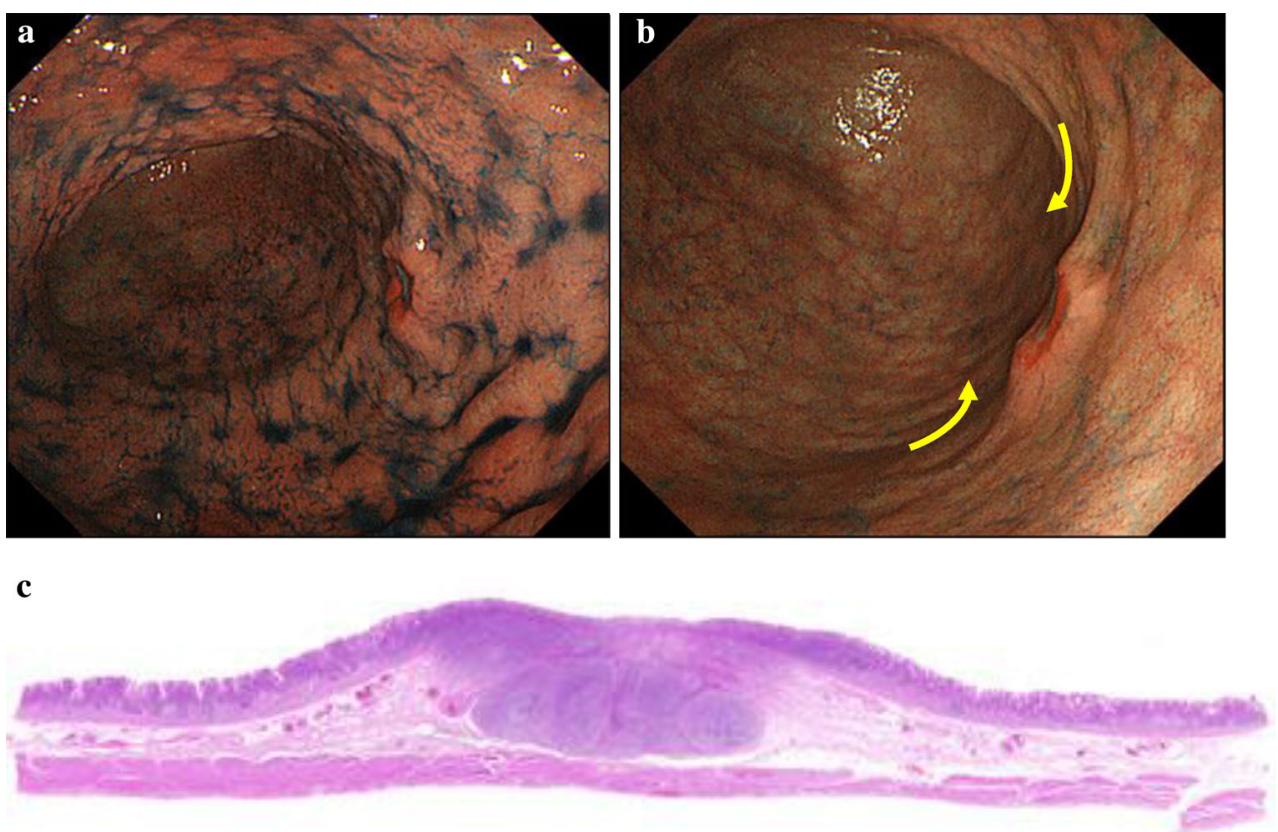
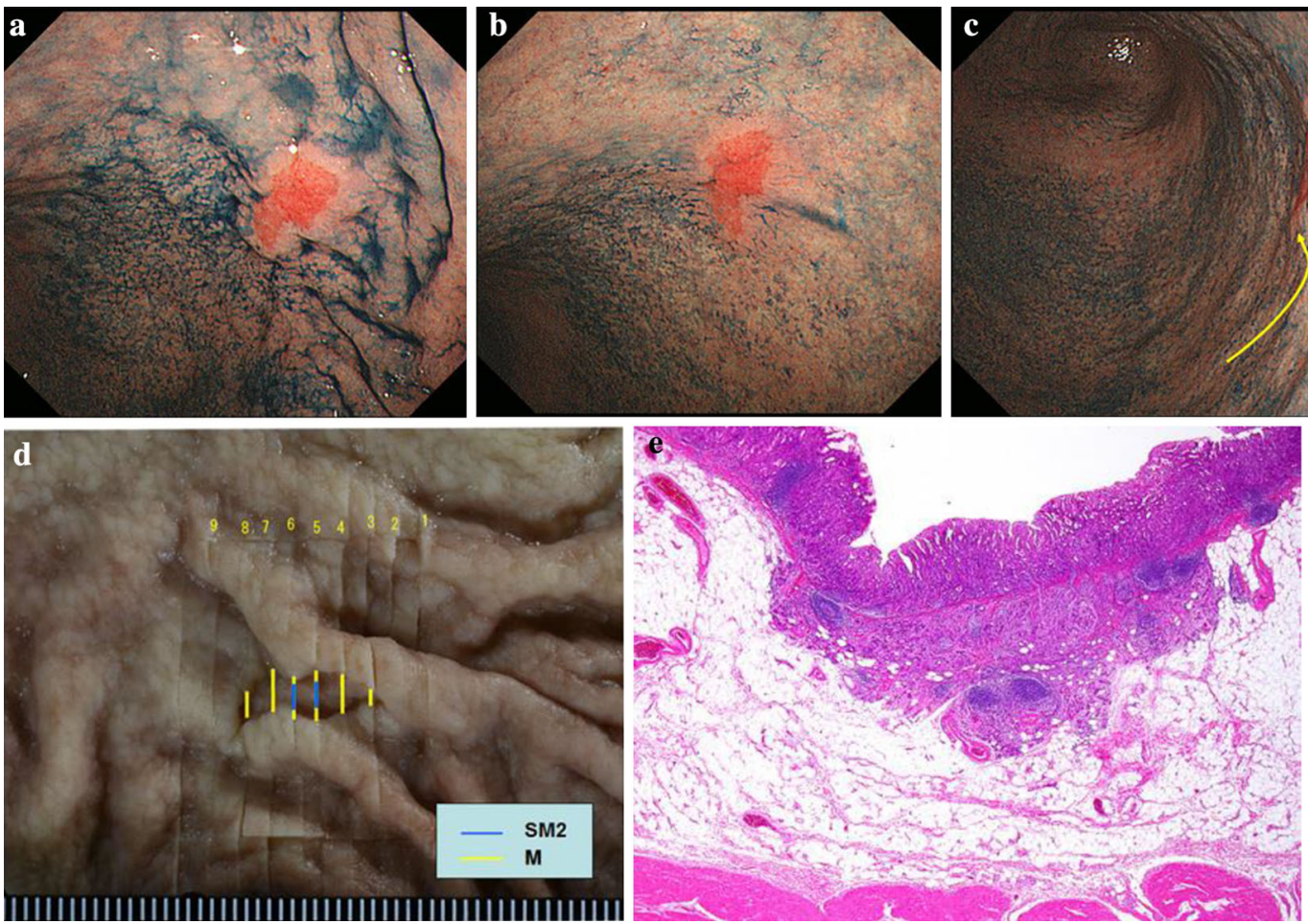

Fig. 3 a 0-IIc type SM2 cancer. With mild extension, the endoscopic findings are of a mildly protruding reddened lesion. b Appearance from directly above following extension of the gastric wall through insufflation of a large volume of air. The entire lesion has become flattened. c Appearance from an angle following extension of the gastric wall through insufflation of a large volume of air. A small nonextension sign has formed in the center of the lesion, with fold convergence and elevation (yellow arrow) in that direction. This lesion was assessed as being positive for the non-extension sign, yielding a diagnosis of SM2 cancer. d Mapping of the histological findings for the lateral extent and depth of invasion in the fixed surgically resected specimen. Blue lines indicate SM2 cancer; yellow lines indicate $\mathrm{M}$ cancer. e Histological findings for slice no. 5. The depth of SM2 invasion of $1700 \mu \mathrm{m}$ yields a diagnosis of SM2invasive cancer 


\section{Handling of resected specimens and histological diagnosis of depth of invasion}

The resected specimen was placed on a flat board with the mucosal side facing upward, pinned at the edges with stainless steel pins, and fixed in a $20 \%$ buffered formalin solution. For endoscopically resected specimens, the fixed specimen was sectioned serially at 2- to $3-\mathrm{mm}$ intervals. For surgically resected specimens, the fixed specimen was sectioned serially at $2-$ to $5-\mathrm{mm}$ intervals.

Histological diagnosis of the depth of invasion was performed in accordance with the Japanese Gastric Cancer Association's classification of gastric cancer [2]. In other words, when microscopic examination revealed submucosal invasion by cancer cells, we measured the vertical distance from the inferior margin of the muscularis mucosae to the deepest point of the invading cancer. If the muscularis mucosae could not be identified due to the presence of ulceration or an ulcer scar, we made the measurement using an imaginary line that continued from the adjacent normal muscularis mucosae. We classified lesions with a maximum vertical invasion depth of less than $500 \mu \mathrm{m}$ as SM1, and those with a maximum vertical invasion depth of $500 \mu \mathrm{m}$ or more as SM2. These histopathological diagnoses were used as the gold standard.

\section{Outcome measurements}

\section{Evaluation of diagnostic ability}

Lesions diagnosed both endoscopically and histologically as SM2 were classified as true-positive lesions (Figs. 2a-c, 3a-e). Lesions diagnosed endoscopically as SM2 but histologically as M or SM1 were classified as false-positive lesions.

Lesions diagnosed endoscopically as M-SM1 and histologically as M or SM1 were classified as true-negative lesions (Fig. 4a-d).

Lesions diagnosed endoscopically as M-SM1 and histologically as SM2 were classified as false-negative lesions.

We determined the frequency of each type of lesion and assessed the sensitivity, specificity, positive predictive value, negative predictive value, and overall accuracy.

\section{Evaluation of diagnostic limitations}

We compared tumor diameters, macroscopic types (elevated or depressed/flat), ulceration findings (absence or presence), location (upper, middle, lower, or residual stomach), histological type (differentiated type or undifferentiated type), and SM2 invasion depth (500-999, 1000-1999 or $2000 \mu \mathrm{m}$ or more) between true-positive and
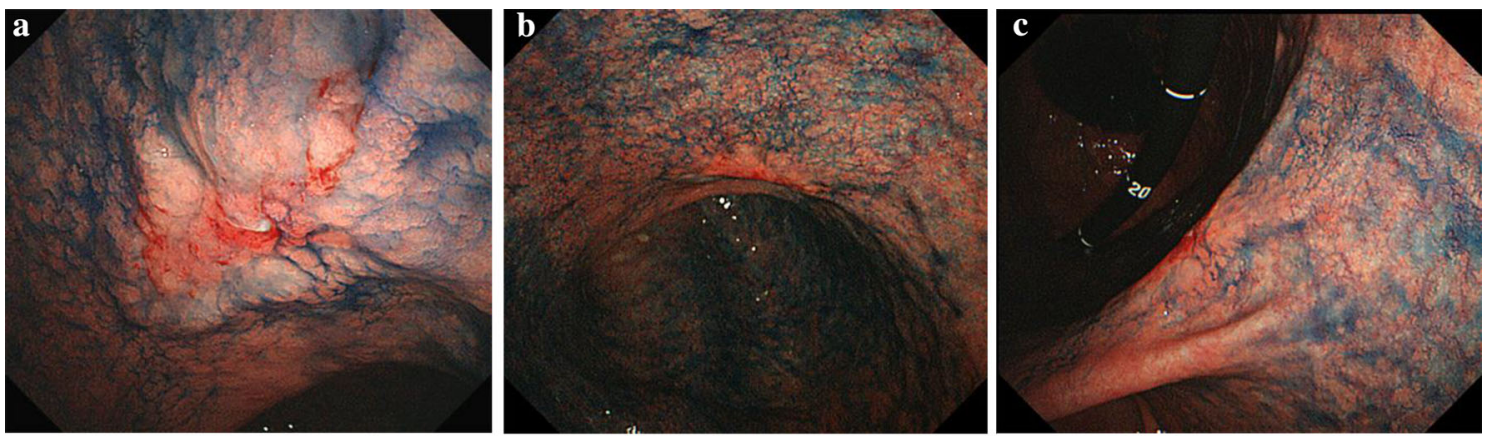

d

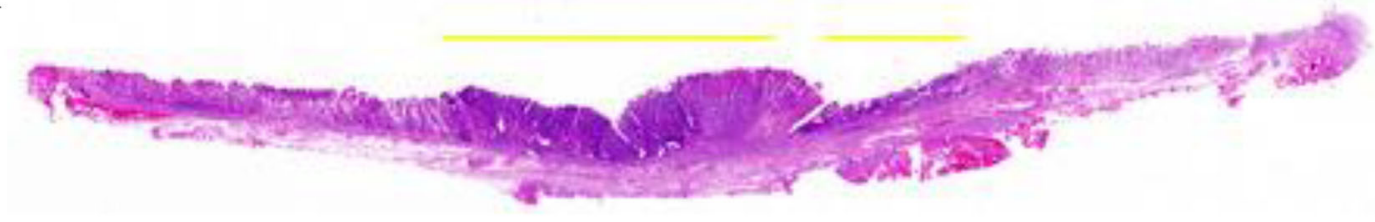

\section{Cancer}

Fig. 4 a 0-IIa + IIc mucosal cancer with an ulcer scar. With mild extension of the gastric wall, the endoscopic findings are of a protruding lesion with converging folds. b With strong extension of the gastric wall through insufflation of a large volume of air, the entire lesion becomes flattened. c Examination from a tangential direction. No "massive elevation" has formed, and the converging mucosal folds can be seen to head for the center of the ulcer scar, with no elevation. This lesion was assessed as being negative for the nonextension sign, yielding a diagnosis of M-SM1 cancer. d The histological findings were of a mucosal cancer associated with an ulcer scar. The level of inflammatory fibrosis in the submucosa gradually becomes coarser as we move from the center of the scar to its periphery (yellow lines: cancer) 
false-negative groups in cases of pathological SM2 cancer. We also compared clinicopathological characteristics between the true-negative and false-positive groups in cases of pathological M-SM1 cancer.

\section{Statistical analysis}

Statistical analyses were performed using the $X^{2}$ test and Fisher's test as appropriate. All significant data are twotailed, and a $P$ value $<0.05$ was considered significant. SPSS version 11.5J software (SPSS, Chicago, IL, USA) was used for all statistical processing.

\section{Results}

\section{Clinicopathological characteristics of early gastric cancers and patients}

From the pathological diagnosis database, 1,055 lesions were sampled from among all gastric cancers endoscopically or surgically resected at Fukuoka University Chikushi Hospital from January 2006 to December 2012. Of these, 185 advanced cancers (including 29 cases of 0 advanced gastric cancers) and two lesions where invasion depth diagnosis was histopathologically difficult were excluded, resulting in the sampling of 868 EGCs. None of these cases had been subject to preoperative endoscopic examination at our hospital. Based on records in the endoscopy database, a further five lesions described as undiagnosable due to poor observation conditions were excluded (three lesions where strong extension of the gastric wall was impossible due to a gag reflex and two lesions where observation was impossible due to bleeding from the lesion). The aforementioned 863 lesions in 704 patients were analyzed in this study. Incidentally, one surgically resected lesion of pathological advanced gastric cancer was clinically diagnosed as M-SM1 during the study period. This lesion was excluded. Furthermore, no surgically resected AGC lesions were pathologically diagnosed as M-SM1 (Fig. 5).

Subjects comprised 494 male and 210 female patients (mean age 69.6 years). Mean tumor size was $21.8 \mathrm{~mm}$, $109,428,313$, and 13 lesions were located in the upper, middle, lower, and residual stomach, respectively. The macroscopic type was elevated in 554 lesions and depressed/flat in 309 lesions. Ulceration was present and absent in 168 and 695 lesions, respectively. Depth of invasion was classed as M, SM1, and SM2 in 655, 87, and 121 lesions, respectively, and the histological type was differentiated and undifferentiated in 727 and 136 lesions, respectively. Six hundred forty-five lesions were endoscopically resected (26 lesions underwent endoscopic resection with additional

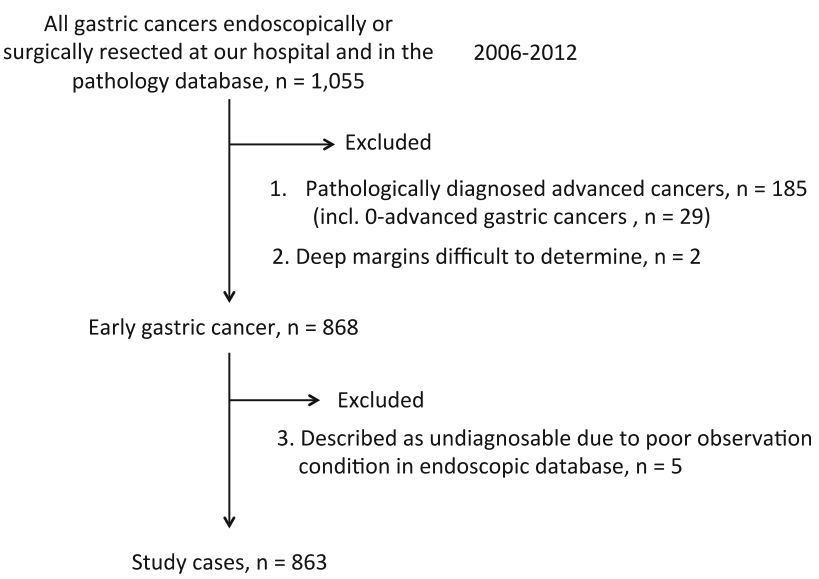

Fig. 5 Sampling of study subjects

surgical resection), and 218 lesions were surgically resected.

\section{Diagnostic ability to determine depth of invasion using the non-extension sign}

There were 104 true-positive, 733 true-negative, 9 falsepositive, and 17 false-negative lesions. This yielded a sensitivity of $92.0 \%$ (95\% confidence interval (CI), 87.0-97.0\%), a specificity of $97.7 \% \quad(95 \% \quad \mathrm{CI}$, 96.7-98.8\%), a positive predictive value of $85.9 \%$ (95\% CI, 79.7-92.1\%), a negative predictive value of $98.8 \%$ (95\% CI, 98.0-99.6\%), and a diagnostic accuracy of $96.9 \%$ (95\% CI, 95.8-98.1\%) (Table 1).

\section{Limitations of diagnosing depth of invasion using the non-extension sign}

We compared the clinicopathological characteristics of the non-extension sign true-positive group of 104 lesions and the non-extension sign false-negative group of 17 lesions among the 124 lesions histologically confirmed as SM2. No significant differences were seen between the groups in terms of tumor size, macroscopic type, ulcer finding, location, or histological type. Classifying the lesions into three groups by

Table 1 Diagnostic performance of conventional endoscopy in the determination of the depth of invasion by early gastric cancer

\begin{tabular}{llrc}
\hline & & \multicolumn{2}{c}{ Histological diagnosis } \\
\cline { 3 - 4 } & & SM2 & M-SM1 \\
\hline Endoscopic diagnosis & SM2 & 104 & 9 \\
& M-SM1 & 17 & 733 \\
\hline
\end{tabular}

SM2 deeper submucosal cancer, $M$-SM1 mucosal cancer and superficial submucosal cancer 
Table 2 Clinicopathological characteristics of the falsenegative group

\begin{tabular}{|c|c|c|c|}
\hline & True-positive group $(n=104)$ & False-negative group $(n=17)$ & $p$ value \\
\hline \multicolumn{4}{|l|}{ Tumor size (mm) } \\
\hline$\sim 10$ & 9 & 1 & 0.575 \\
\hline $11-20$ & 21 & 1 & 0.156 \\
\hline$\geq 21$ & 74 & 15 & 0.139 \\
\hline \multicolumn{4}{|l|}{ Macroscopic type } \\
\hline Elevated & 34 & 3 & 0.266 \\
\hline Depressed/flat & 70 & 14 & 0.266 \\
\hline \multicolumn{4}{|l|}{ Ulceration finding } \\
\hline Presence & 14 & 5 & 0.094 \\
\hline Absence & 90 & 12 & 0.094 \\
\hline \multicolumn{4}{|l|}{ Location } \\
\hline Upper third & 16 & 4 & 0.480 \\
\hline Middle third & 52 & 10 & 0.604 \\
\hline Lower third & 34 & 3 & 0.266 \\
\hline Residual stomach & 2 & 0 & 1.000 \\
\hline \multicolumn{4}{|l|}{ Histological type } \\
\hline Differentiated & 70 & 12 & 1.000 \\
\hline Undifferentiated & 34 & 5 & 1.000 \\
\hline \multicolumn{4}{|l|}{ SM2 depth $(\mu \mathrm{m})$} \\
\hline $500-999$ & 22 & 12 & $<0.001 *$ \\
\hline 1000-1999 & 36 & 2 & 0.089 \\
\hline$\geq 2000$ & 46 & 3 & 0.060 \\
\hline
\end{tabular}

$\chi^{2}$ test, Fisher's test: $*$ indicates significance the vertical depth of SM invasion yielded true-positive rates of $65 \%(22 / 34)$ in the 500-999 $\mu \mathrm{m}$ group, $95 \%(36 / 38)$ in the 1000-1999 $\mu \mathrm{m}$ group, and $94 \%(46 / 49)$ in the $\geq 2000$ $\mu \mathrm{m}$ group. The false-negative rate was therefore significantly greater in the 500-999 $\mu \mathrm{m}$ group (Tables 2, 3).

We then compared the clinicopathological characteristics of the non-extension sign true-negative group of 733 lesions and the non-extension sign false-positive group of 9 lesions among the 742 lesions histologically confirmed as M-SM1. No significant differences were seen between the groups in macroscopic type, location, or histological type. Classifying lesions into three groups by tumor size yielded true-negative rates of $100 \%$ (256/256) in the $\leq 10$-mm group, $99.2 \%$ (244/ 246 ) in the 11- to 20 -mm group, and $97.0 \%$ (233/240) in the $\geq 21$-mm group. The false-positive rate increased as the tumor size increased (Table 3). True-negative rates according to ulcer findings were $99.3 \%$ (629/633) for absence and $95.4 \%$ $(104 / 109)$ for absence. The false-positive rate was therefore higher for the presence of ulcer findings (Table 3).

\section{Discussion}

Accurate diagnosis of the depth of invasion is one of the most important factors needed to determine the chances of a successful curative procedure when deciding upon the therapeutic regimen for EGC. Endoscopic ultrasonography (EUS) can be considered the standard method for determining the depth of invasion by EGCs. However, even recent studies have reported widely varying diagnostic accuracies of $67-90 \%$ when determining the depth of invasion using EUS, making it difficult to view the method as sufficiently reliable [9-14]. Furthermore, its diagnostic ability is similar to that of CE alone [15-17], and a number of studies have stated that EUS is unsuitable as a routine investigation for determining the depth of invasion by EGCs [15]. In recent years, there have been some promising reports regarding the determination of invasion depth using new modalities such as infrared endoscopy [18] and magnifying endoscopy with narrow-band imaging [19], but these are yet to produce consistently reliable results.

Yao et al. [8] analyzed the incidence of the non-extension sign in SM1 and SM2 cancers, reporting that (1) the non-extension sign is not seen in SM1 cancers; (2) in histologically confirmed SM2 lesions, the incidence of the non-extension sign is $100 \%$ in lesions with a vertical SM invasion depth of $1600 \mu \mathrm{m}$ or more; and (3) the incidence of the non-extension sign is $86 \%$ in lesions with a vertical invasion depth of 500-1599 $\mu \mathrm{m}$ if the lateral extent of invasion is $\geq 2600 \mu \mathrm{m}$. Furthermore, they reported that the false-positive rate is high if the non-extension sign is 
Table 3 Clinicopathological characteristics of the falsepositive group

\begin{tabular}{llll}
\hline & True-negative group $(n=733)$ & False-positive group $(n=9)$ & $p$ value \\
\hline Tumor size $(\mathrm{mm})$ & & & \\
$\sim 10$ & 256 & 2 & $0.028^{*}$ \\
$11-20$ & 244 & 7 & 0.381 \\
$\geq 21$ & 233 & & $0.007^{*}$ \\
Macroscopic type & & 5 & 0.300 \\
Elevated & 267 & 4 & 0.300 \\
Depressed/flat & 466 & & \\
Ulceration finding & & 4 & $0.005^{*}$ \\
Absence & 629 & 5 & $0.005^{*}$ \\
Presence & 104 & & \\
Location & & 1 & 0.700 \\
Upper third & 89 & 6 & 0.237 \\
Middle third & 359 & 2 & 0.287 \\
Lower third & 274 & 0 & 1.000 \\
Residual stomach & 11 & & 0.335 \\
Histological type & & 7 & 0.335 \\
Differentiated & 638 & 2 & \\
Undifferentiated & 95 & & \\
\hline
\end{tabular}

Fisher's test: * indicates significance assessed when the gastric wall has not been extended with a large volume of air, whereas the false-negative rate is high if the lesion is not examined from an oblique or tangential direction [8]. Strong extension of the gastric wall is defined as strong extension that meets one or both of the following criteria: (1) full extension of the folds on the greater curvature of the gastric corpus; (2) extension until the blood vessels surrounding the EGC can be clearly delineated. All endoscopists determined the presence or absence of the non-extension sign based on these criteria for strong extension of the gastric wall.

Furthermore, it was vital to observe the lesion from an oblique or tangential direction if at all possible when determining the presence or absence of the non-extension sign, as this resolves any potential visual issues. Height differences in the lesion are difficult to ascertain when observed from a direction close to a frontal view (Fig. 3b). On the other hand, height differences are much more readily discerned when the lesion is observed from the side (Fig. 3c). Because the non-extension sign protrudes morphologically, observation from a more oblique or tangential direction is needed to accurately perceive subtle changes.

The current study assessed the diagnostic ability of CE using only the non-extension sign under a unified viewing method to determine the depth of invasion by EGCs in a large number of consecutively collected cases. Our results yielded a sensitivity of $92.0 \%$, a specificity of $97.7 \%$, a positive predictive value of $85.9 \%$, a negative predictive value of $98.7 \%$, and a diagnostic accuracy of $96.9 \%$.
Sensitivity, positive predictive value, and diagnostic accuracy were all higher than in previous reports, and the specificity and negative predictive value were particularly high, suggesting high diagnostic ability.

There are three likely reasons why our results were excellent compared with past reports. The first is that previously reported diagnostic markers of invasion depth, such as tumor diameter, macroscopic type, and coloration $[4,5,6,7]$, are markers based on statistical correlations that are independent of histopathological features. In contrast, the non-extension sign is a marker with a direct causal link to the histopathological features of SM2 invasion, which suggests high sensitivity and specificity. The second reason is that we performed diagnoses under fixed observation conditions in order to accurately determine the presence or absence of the non-extension sign, which may explain why diagnostic objectivity and constancy were maintained among all endoscopists. The third reason is the importance of observing the region of interest from an oblique or tangential direction for accurate determination of the presence or absence of the non-extension sign. We used a side-viewing gastroscope that is considered to have excellent intragastric observation capabilities in almost all cases of EGC diagnosed by CE. This may explain why we were able to accurately discern minute findings.

Reported limitations of EUS - which is widely used to diagnose depth of invasion by EGCs-include lesions with associated ulceration, lesions located in the upper stomach, and lesions with a large diameter [9-14]. 
To determine the limitations of diagnosis of the depth of invasion using the non-extension sign, we compared the clinicopathological characteristics of non-extension sign true-positive and false-negative groups of patients with lesions that were histologically confirmed as SM2. The results showed that the false-negative rate was significantly higher in the group of lesions with a vertical SM invasion depth of 500-999 $\mu \mathrm{m}$, indicating a limitation of this method. We can speculate that the mild degree of thickening and hardening of the submucosa in this 500-999 $\mu \mathrm{m}$ group prevents the formation of an endoscopically identifiable non-extension sign. In clinical practice, these lesions would be resected endoscopically. Histological examination of the resected specimen will indicate the necessity for further surgical treatment, with no adverse effects on patient prognosis.

A similar comparison of the clinicopathological characteristics of the non-extension sign true-negative and false-positive groups of patients with histologically confirmed M-SM1 lesions revealed that the false-positive rate increased with increasing tumor size, and was higher for the ulcerated type. This suggests that careful examination, including another modality, may be warranted for larger lesions and lesions with associated ulceration.

The limitations of this study are the single-center retrospective study design, and the fact that a comparison with the diagnostic ability of EUS was not undertaken. In an earlier report, we compared the accuracy of CE for diagnosing invasion depth with that of EUS using just the non-extension sign [20]. We extracted 341 non-consecutive lesions of EGC, the respective sensitivity, specificity, and accuracy of CE compared to EUS were $89.5 \%(95 \% \mathrm{CI}$, 81.5-97.4\%) vs. $68.4 \% \quad(95 \%$ CI, $56.3-80.5 \%$; $p=0.01), 99.3 \%(98.3-100 \%)$ vs. $83.5 \%(79.1-87.8 \%$; $p<0.0001)$, and $98.4 \% \quad(96-99.3 \%) \quad$ vs. $80.9 \%$ (76.8-85.1\%; $p<0.0001)$. This indicated that CE had superior diagnostic accuracy [20]. The fact that high specificity and negative predictive values were obtained in the present study suggests that EUS can be dismissed in favor of endoscopic therapy for lesions deemed negative for the non-extension sign under highly accurate examination methods. However, because this was a retrospective study of nonconsecutive patients randomly sampled from cases of EUS, a multicenter prospective controlled trial should now be conducted.

In conclusion, the results of this study indicate that the non-extension sign, a diagnostic criterion that can be assessed using CE, may offer a straightforward and effective means of accurately determining the suitability of minimally invasive endoscopic treatment. However, considering the limitations of retrospective analysis, a further prospective study is warranted to confirm the diagnostic reliability of the non-extension sign.
Acknowledgments This study was not funded by any grants.

\section{Compliance with ethical standards}

Conflict of interest The authors declare no conflict of interest for this article.

Ethical standard This study was approved by the Medical Ethics Committee of Fukuoka University Chikushi Hospital.

\section{References}

1. Gotoda T, Yanagisawa A, Sasako M, et al. Incidence of lymph node metastasis from early gastric cancer: estimation with a large number of cases at two large centers. Gastric Cancer. 2000;3:219-25.

2. Sano T, Kodera Y. Japanese classification of gastric carcinoma: 3rd English edition. Gastric Cancer. 2011;14:101-12.

3. Japanese Gastric Cancer Association. Japanese classification of gastric carcinoma-2nd English edition. Gastric Cancer. 1998;1:10-24.

4. Sano T, Okuyama Y, Kobori O, et al. Early gastric cancer. Endoscopic diagnosis of depth of invasion. Dig Dis Sci. 1990;35:1340-4.

5. Namieno T, Koito K, Higashi T, et al. Endoscopic prediction of tumor depth of gastric carcinoma for assessing the indication of its limited resection. Oncol Rep. 2000;7:57-61.

6. Abe S, Oda I, Shimazu T, et al. Depth-predicting score for differentiated early gastric cancer. Gastric Cancer. 2011;14:35-40.

7. Choi J, Kim SG, Im JP, et al. Endoscopic prediction of tumor invasion depth in early gastric cancer. Gastrointest Endosc. 2009;73:917-27.

8. Yao T, Tanabe H, Nagahama T, et al. Clinicopathological study for accurate endoscopic diagnosis of submucosal invasion by early gastric cancer of depressed type. Stomach Intest (Tokyo). 2008;43:1109-25 (in Japanese, with English abstract).

9. Mandai K, Yasuda K. Accuracy of endoscopic ultrasonography for determining the treatment method for early gastric cancer. Gastroenterol Res Pract. 2012;2012:245390.

10. Yamamoto S, Nishida T, Kato M, et al. Evaluation of endoscopic ultrasound image quality is necessary in endosonographic assessment of early gastric cancer invasion depth. Gastroenterol Res Pract. 2012;2012:194930.

11. Tsuzuka T, Okada H, Kawahara Y, et al. Usefulness and problems of endoscopic ultrasonography in prediction of the depth of tumor invasion in early gastric cancer. Acta Med Okayama. 2011;65:105-12.

12. Akahoshi K, Chijiwa Y, Hamada S, et al. Pretreatment staging of endoscopically early gastric cancer with a $15 \mathrm{MHz}$ ultrasound catheter probe. Gastrointest Endosc. 1998;48:470-6.

13. Mouri R, Yoshida S, Tanaka S, et al. Usefulness of endoscopic ultrasonography in determining the depth of invasion and indication for endoscopic treatment of early gastric cancer. Clin $\mathrm{J}$ Gastroenterol. 2009;43:318-22.

14. Kim JH, Song KS, Youn YH, et al. Clinicopathologic factors influence accurate endosonographic assessment for early gastric cancer. Gastrointest Endosc. 2007;66(5):901-8.

15. Choi J, Kim SG, Im JP, et al. Comparison of endoscopic ultrasonography and conventional endoscopy for prediction of depth of tumor invasion in early gastric cancer. Endoscopy. 2010;42:705-13.

16. Hizawa K, Iwai K, Ezaki M, et al. Is endoscopic ultrasonography indispensable in assessing the appropriateness of endoscopic resection for gastric cancer? Endoscopy. 2002;34:973-8. 
17. Yanai H, Noguchi T, Mizumachi S, et al. A blind comparison of the effectiveness of endoscopic ultrasonography and endoscopy in staging early gastric cancer. Gut. 1999;44:361-5.

18. Ishihara R. Infrared endoscopy in the diagnosis and treatment of early gastric cancer. Endoscopy. 2010;42:672-6.

19. Kikuchi D, Iizuka T, Hoteya S, et al. Usefulness of magnifying endoscopy with narrow-band imaging for determining tumor invasion depth in early gastric cancer. Gastroent Res Pract. 2013;2013:1-5.

20. Nagahama T, Otsu K, Yao K, et al. Conventional endoscopy using "non-extension sign" as a diagnostic marker is superior to endoscopic ultrasonograpy (EUS) for diagnosing depth of invasion in early gastric cancer. United Eur Gastroenterol J. 2014;2(Suppl 1):A481. 